\title{
Prostate-specific membrane antigen (PSMA) expression in adenoid cystic carcinoma of the head and neck
}

Thomas J. W. Klein Nulent ${ }^{1,2^{*}}$, Matthijs H. Valstar ${ }^{3,4}$, Laura A. Smit ${ }^{5}$, Ludwig E. Smeele ${ }^{3,4}$, Nicolaas P. A. Zuithoff ${ }^{6}$, Bart de Keizer ${ }^{7}$, Remco de Bree ${ }^{1}$, Robert J. J. van $\mathrm{Es}^{1,2}$ and Stefan M. Willems ${ }^{8}$

\begin{abstract}
Background: Treatment options for advanced head and neck adenoid cystic carcinoma (AdCC) are limited. Prostate-Specific Membrane Antigen (PSMA), a transmembrane protein that is known for its use in diagnostics and targeted therapy in prostate cancer, is also expressed by AdCC. This study aimed to analyse PSMA expression in a large cohort of primary, recurrent and metastasized AdCC of the head and neck.

Methods: One hundred ten consecutive patients with histologically confirmed AdCC in the period 1990-2017 were included. An analysis was made of clinical details, revised pathology and semiquantitative immunohistochemical expression of PSMA on tissue microarray and whole slides. Associations of PSMA expression with clinicopathological parameters were explored and survival was analysed by multivariate Cox-proportional Hazard analysis.

Results: PSMA expression was present in 94\% of the 110 primary tumours, with a median of $31 \%$ positive cells (IQR 15-60\%). Primary tumours $(n=18)$ that recurred $(n=15)$ and/or had metastases $(n=10)$ demonstrated 40, 60 and $23 \%$ expression respectively. Expression was not independently related to increased pathological stage, tumour grade, and the occurrence of locoregional recurrence or metastasis. After dichotomization, only primary tumour PSMA expression $\leq 10 \%$ appeared to be associated with reduced 10-years recurrence-free survival $(\mathrm{HR} 3.0,95 \% \mathrm{Cl}$ $1.1-8.5, p=.04)$.

Conclusions: PSMA is highly expressed in primary, recurrent and metastatic AdCC of the salivary and seromucous glands. PSMA expression has no value in predicting clinical behaviour of AdCC although low expression may indicate a reduced recurrence-free survival. This study provides supporting results to consider using PSMA as target for imaging and therapy when other diagnostic and palliative treatment options fail.
\end{abstract}

Keywords: Adenoid cystic carcinoma, Salivary gland neoplasms, Immunohistochemistry, Survival analysis, PSMA, Prostate-specific membrane antigen

\footnotetext{
*Correspondence: t.j.w.kleinnulent@umcutrecht.nl

'Department of Head and Neck Surgical Oncology, UMC Utrecht Cancer Center, University Medical Center Utrecht, Heidelberglaan 100, P.O. Box 85500, Utrecht 3508, GA, The Netherlands

${ }^{2}$ Department of Oral and Maxillofacial Surgery, University Medical Center Utrecht, Utrecht, The Netherlands

Full list of author information is available at the end of the article
}

(C) The Author(s). 2020 Open Access This article is licensed under a Creative Commons Attribution 4.0 International License, which permits use, sharing, adaptation, distribution and reproduction in any medium or format, as long as you give appropriate credit to the original author(s) and the source, provide a link to the Creative Commons licence, and indicate if changes were made. The images or other third party material in this article are included in the article's Creative Commons licence, unless indicated otherwise in a credit line to the material. If material is not included in the article's Creative Commons licence and your intended use is not permitted by statutory regulation or exceeds the permitted use, you will need to obtain permission directly from the copyright holder. To view a copy of this licence, visit http://creativecommons.org/licenses/by/4.0/ The Creative Commons Public Domain Dedication waiver (http://creativecommons.org/publicdomain/zero/1.0/) applies to the data made available in this article, unless otherwise stated in a credit line to the data. 


\section{Background}

The Prostate-specific membrane antigen (PSMA) is a transmembrane glycoprotein of the prostate secretory acinar epithelium that is upregulated in prostate cancer (PC) and known from its use in diagnostics and targeted therapy in metastatic PC [1-4]. Besides tracer accumulation in prostate tissue, PSMA PET/CT depicts physiological uptake in the salivary and lacrimal glands, liver and kidneys, but also in benign and malignant neoplasms, mostly adenomas and (adeno) carcinomas, of glandular or epithelial origin $[5,6]$.

In PC, increased intracellular PSMA expression by immunohistochemistry is related to increased pathological grade, and subsequently correlated with disease-related mortality [1-4]. Malignancies other than PC also express PSMA but in endothelial cells of tumours' neovasculature, which suggests PSMA involvement in tumour angiogenesis. In salivary glands PSMA was identified on the acinar cells in the epithelium [3, 7-9].

Recently, PSMA PET/CT analysis in a series of patients with head and neck adenoid cystic carcinoma (AdCC) showed tracer uptake in areas of locoregional recurrent and distant metastatic AdCC, and expression was confirmed immunohistochemically [10]. AdCC is the most common malignant secretory gland tumour in the head and neck region. Incidence peaks in the fifth and sixth decade and has a female predominance [1115]. AdCC originates from ductal (luminal) and basal/ myoepithelial (abluminal) cells and typically arises in the major salivary glands, the minor salivary and seromucous glands of the lip and upper aerodigestive tract, but also in the lacrimal and ceruminous glands. The tumour is characterized by an indolent but persistent growth rate, frequent locoregional recurrence and a delayed silent onset of distant metastasis, mainly in the lungs [11, 15-17]. Surgery is the primary treatment, frequently followed by adjuvant radiation therapy because of positive resection margins and typical perineural growth. Although radiotherapy has probably no benefit to survival, it is reported to improve local and regional control [15, 16]. Disease-specific survival (DSS) is moderate, with five and 10 year survival rates of $68-78 \%$ and $54-65 \%$ respectively $[18,19]$. Survival is negatively affected by the occurrence of an irresectable locoregional recurrence, which is considered clinically more relevant than the occurrence of slowly growing -often pulmonary and osseous- distant metastases that develop in almost half of the patients within 5 years after diagnosis [11, 16, 18]. Other negative prognostic factors are advanced tumour stage, inadequate resection margins, skull base involvement and a solid growth pattern on histopathology. Perineural invasion does not directly affect mortality, but is significantly correlated with metastatic disease $[11,15]$. Regular treatment options are limited in advanced recurrent or metastasized disease [16]. Given the positive results of PSMA-targeted diagnostic and treatment modalities in $\mathrm{PC}$, this study aimed to analyse PSMA expression in a large cohort of primary and corresponding recurrent and metastatic AdCC tissues of the head and neck [20]. Secondly, we aimed to explore associations with patient- and tumour characteristics and outcome, analogous to PC.

\section{Methods}

\section{Patient selection}

All patients diagnosed with AdCC in the University Medical Center Utrecht and Netherlands Cancer Institute-Antoni van Leeuwenhoek Hospital between 1990 and 2017 were analysed in a retrospective cohort study. Patients were selected in case of a histologyproven primary AdCC in the head and neck region with available representative formaldehyde-fixed paraffinembedded tissue blocks of the resection specimen. Tumour samples of available corresponding locoregional recurrences or distant metastases were collected. Patients with previous (non AdCC) salivary gland disease, radiotherapy to the head or neck, or incomplete data were excluded. All data and samples were handled according to the GDPR.

\section{Clinical parameters and tumour characteristics}

The following clinical parameters were retrieved from the medical files: patient's gender, age at diagnosis, tumour site, treatment regimen, (time to) recurrence or metastasis, vital status (cause of death) and date of last follow-up until January 1st 2018. Two dedicated head and neck pathologists (S.W. and L.S.) re-examined all haematoxylin- and eosin-stained slides for the following parameters: type and diameter of the tumour, pathological $\mathrm{T}$ - and $\mathrm{N}$-stage, histopathological growth pattern and associated grade according to the differentiation of Perzin et al. [21], surgical resection margins and the presence of perineural, vascular and bone invasion.

\section{PSMA immunohistochemistry}

A Tissue Microarray (TMA) was used to assess PSMA expression. From each tumour, three central $0.6 \mathrm{~mm}$ tissue cylinders from vital tumour were incorporated and covered the different aspects of this tumour morphology. Tumour whole-slides were analysed when patient's tissue was not incorporated in the microarray, as well as whole-slides of all available recurrent and distant tissues. Representative TMA or whole slide paraffin sections $4 \mu \mathrm{m}$ thick were immunohistochemically stained using fully automated protocols on the Benchmark XT (Ventana Medical Systems, Tucson, AZ, USA), validated for diagnostic purposes. Incorporated as control tissues were prostate cancer, normal salivary gland and duct tissue. 
For the primary antibody, a mouse antihuman PSMA monoclonal antibody was used (3E6; DAKO, Carpinteria, CA) of the IgG1 isotype directed against the internal domain of the PSMA antigen (DAKO, cat. no. M3620, Carpinteria, CA, dilution 1/80). The tissue sections were deparaffinised with xylene and ethanol followed by Heat Induced Epitope Retrieval in Ventana Cell Conditioning 1 for $24 \mathrm{~min}$ and subsequently incubated with the primary antibody for $60 \mathrm{~min}$. Antigen-antibody reactions were visualized using Ventana OptiViewTM Amplification kit, followed by Ventana OptiViewTM Universal DAB Detection Kit (Optiview HQ Linker 8 min, Optiview HRP Multimer 8 min, Optiview Amplifier H2O2/ Amplifier $4 \mathrm{~min}$, Optiview Amplifier Multimer $4 \mathrm{~min}$ ). Finally the slides were counterstained with haematoxylin, dehydrated and mounted.

\section{PSMA expression analysis}

Blinded semiquantitative scoring of all selected primary, recurrent and distant AdCC tumour samples was done until consensus was reached by two head and neck pathologists and two researchers (S.W., L.S., T.K.N. and M.V.). Per tumour core or whole slide the localization of PSMA-positive tumour cells was noted, followed by scoring the percentage of positive tumour cells in increments of 5\%. Total tumour PSMA expression of the arrayed cores was defined by the mean percentage of PSMA-positive tumour cells of the three tissue cores. A core was considered inadequate when it contained $<5 \%$ tumour tissue. In case of a mean PSMA expression below $10 \%$ or in case of more than 1 inadequate core on microarray, one representative tumour whole slide was subsequently stained and scored in order to exclude false-negative results.

\section{Statistical evaluation}

Continuous and ordinal variables were reported as medians with interquartile ranges (IQR), categorical variables were reported as the number of patients and percentages. Associations between PSMA expression and all clinical parameters and tumour characteristics, except when categorical with more than 3 categories, were estimated with Spearman- $\rho$ correlation coefficient with corresponding $p$-values. The Independent Samples Kruskal-Wallis test (KW) was used to compare expression distribution between patients' primary tumours, which recurred or metastasized and those that did not. Subsequently, the median expression of corresponding primary, recurrent and distant samples was compared. A cut-off level for the prediction of overall survival (OS), DSS, locoregional recurrencefree survival (RFS), and metastatic-free survival (MFS) was determined by dichotomizing PSMA expression and plotting Receiver Operating Characteristic (ROC)curves. Differences in baseline characteristics of the groups divided by dichotomization were compared using Pearson chi-square test with appropriate Bonferroni correction. Statistics were performed using SPSS Statistics (version 22.0, IBM Corp., Armonk, NY, USA) for Windows. Multivariate OS, DSS, RFS and MFS survival analyses were carried out to calculate Hazard ratios with 95\% confidence interval (CI). A Cox-proportional Hazard regression model was created by using SAS software (version 9.4, SAS Institute Inc., Cary, NC, USA) for Windows. Firth's correction was applied to reduce bias of maximum likelihood estimation, as it deals with the occurrence of monotone likelihood in small-sample studies with timedependent effects [22]. Discriminative ability of the model was assessed by computing Harrell's C-statistic [23]. A two-tailed $P$-value $<0.05$ was considered statistically significant for all analyses.

\section{Results}

Patients, clinical parameters and tumour characteristics

In total 122 newly diagnosed patients with AdCC of the head and neck were identified within the 27-year period, of whom 12 were excluded because of incomplete data. From 110 patients the clinical history and histopathological samples of the primary tumour were available for analysis and summarized in Table 1.

\section{PSMA immunohistochemistry}

Samples of 73 out of 110 patients were available for TMA immunohistochemistry. Of the remaining 37 primary tumours and available locoregional $(n=15)$ and distant $(n=10)$ tumour samples whole-slides were separately stained and scored. Expression was seen intracellular in a granular fashion, mainly cytoplasmic, or concentrated at the luminal side of the cell membrane. No conclusive staining pattern within the cells was observed. The staining intensity was consistent and there was limited spatial variability. Staining of the whole slides and microarray cores were comparable and therefore usage of this TMA was considered reliable. TMA analysis of 14 tumours was unsuccessful due to inadequate cores, and $21 \mathrm{tu}-$ mours initially scored $0-10 \%$ expression. Matched whole slides of these 35 tumours were subsequently stained and PSMA expression was adjusted in six cases with conflicting ( $>10 \%$ difference) results. Different immunohistochemistry examples are visualized in Fig. 1.

PSMA expression analysis in primary AdCC

In 103 out of 110 primary tumours (94\%) PSMA was expressed with median $31 \%$ positive tumour cells (IQR 
Table 1 Cohort Characteristics

\begin{tabular}{|c|c|c|c|c|c|c|}
\hline & $\mathrm{N}(\%)$ & Median \% PSMA & $\begin{array}{l}\text { Spearman- } \rho ; \\
p \text {-value }\end{array}$ & PSMA $\leq 10 \%$ & PSMA>10\% & $\begin{array}{l}\text { Chi }^{2} \text { PSMA } \\
\leq 10 \% \text { vs }>10 \%\end{array}$ \\
\hline Patients & 110 & $31 \%$ & & $23(21 \%)$ & 87 (79\%) & \\
\hline \multicolumn{7}{|l|}{ Gender } \\
\hline Male & $36(33 \%)$ & $30 \%$ & $.11 ; p=.24$ & $7(19 \%)$ & $29(81 \%)$ & $p=.79$ \\
\hline Female & $74(67 \%)$ & $45 \%$ & & $16(22 \%)$ & $58(78 \%)$ & \\
\hline \multicolumn{7}{|l|}{ Age at diagnosis } \\
\hline Median (IQR) & $57(45-68)$ & & $.08 ; p=.42$ & $56(50-70)$ & $58(43-68)$ & $p=.45$ \\
\hline Range & $20-90$ & & & $34-87$ & $20-90$ & \\
\hline \multicolumn{7}{|l|}{ Site and subsite } \\
\hline aMajor salivary gland & 59 (54\%) & $50 \%$ & $-.25 ; p<.01$ & $7(12 \%)$ & $52(88 \%)$ & $p=.01$ \\
\hline Parotid gland & 26 & $50 \%$ & & 2 & 24 & $p=.06$ \\
\hline Submandibular gland & 30 & $35 \%$ & & 5 & 25 & $p=.50$ \\
\hline Sublingual gland & 3 & $57 \%$ & & 0 & 3 & $p=.37$ \\
\hline${ }^{\mathrm{a}}$ Minor salivary and seromucous gland & $51(46 \%)$ & $21 \%$ & & $16(31 \%)$ & $35(69 \%)$ & \\
\hline $\begin{array}{l}\text { Oral cavity (lip/buccal mucosa/hard } \\
\text { palate/gum) }\end{array}$ & 21 & $19 \%$ & & 7 & 14 & $p=.12$ \\
\hline Oropharynx (soft palate/base of tongue) & 8 & $21 \%$ & & 1 & 7 & $p=.54$ \\
\hline Nasal cavity/nasopharynx/maxillary sinus & 15 & $10 \%$ & & 8 & 7 & $p<.01$ \\
\hline Larynx/trachea & 3 & $42 \%$ & & 0 & 3 & $p=.37$ \\
\hline Lacrimal gland & 2 & $35 \%$ & & 0 & 2 & $p=.46$ \\
\hline External auditory canal & 2 & $40 \%$ & & 0 & 2 & $p=.46$ \\
\hline \multicolumn{7}{|l|}{ Tumour } \\
\hline \multicolumn{7}{|l|}{ pT-stage (TNM 7th ed.) } \\
\hline pT1 & 35 & $50 \%$ & $-.18 ; p=.06$ & 5 & 30 & $p=.24$ \\
\hline pT2 & 39 & $33 \%$ & & 7 & 32 & $p=.57$ \\
\hline pT3 & 5 & $20 \%$ & & 1 & 4 & $p=.96$ \\
\hline pT4a & 23 & $30 \%$ & & 6 & 17 & $p=.49$ \\
\hline pT4b & 8 & $13 \%$ & & 4 & 4 & $p=.04$ \\
\hline \multicolumn{7}{|l|}{ Nodal status } \\
\hline pNO & 99 (90\%) & $33 \%$ & $-.09 ; p=.37$ & $20(20 \%)$ & 79 (80\%) & $p=.58$ \\
\hline $\mathrm{pN+}$ & $11(10 \%)$ & $25 \%$ & & $3(27 \%)$ & $8(73 \%)$ & \\
\hline \multicolumn{7}{|l|}{ Distant metastasis } \\
\hline cMO & 108 & $31 \%$ & $-.01 ; p=.90$ & $23(21 \%)$ & 85 (79\%) & $p=.62$ \\
\hline $\mathrm{cM1}$ & 2 & $36 \%$ & & $0(0 \%)$ & $2(100 \%)$ & \\
\hline \multicolumn{7}{|l|}{ Resection margin } \\
\hline clear (>5 mm) & $20(18 \%)$ & $50 \%$ & $-.08 ; p=.43$ & $2(10 \%)$ & $18(90 \%)$ & $p=.18$ \\
\hline close (1-5 mm) & $4(4 \%)$ & $20 \%$ & & $2(50 \%)$ & $2(50 \%)$ & $p=.14$ \\
\hline positive (< $1 \mathrm{~mm}$ ) & $86(78 \%)$ & $30 \%$ & & $19(22 \%)$ & 67 (78\%) & $p=.56$ \\
\hline \multicolumn{7}{|l|}{ Perineural growth } \\
\hline Present & $76(69 \%)$ & $30 \%$ & $-.02 ; p=.86$ & $14(18 \%)$ & $62(82 \%)$ & $p=.44$ \\
\hline Absent & $32(31 \%)$ & $45 \%$ & & $8(25 \%)$ & $24(75 \%)$ & \\
\hline \multicolumn{7}{|l|}{ Vasoinvasive growth } \\
\hline Present & $17(15 \%)$ & $30 \%$ & $-.05 ; p=.64$ & $5(29 \%)$ & $12(71 \%)$ & $p=.33$ \\
\hline Absent & $90(82 \%)$ & $33 \%$ & & 17 (19\%) & 73 (81\%) & \\
\hline
\end{tabular}


Table 1 Cohort Characteristics (Continued)

\begin{tabular}{|c|c|c|c|c|c|c|}
\hline & N (\%) & Median \% PSMA & $\begin{array}{l}\text { Spearman-p; } \\
p \text {-value }\end{array}$ & PSMA $\leq 10 \%$ & PSMA $>10 \%$ & $\begin{array}{l}\text { Chi }^{2} \text { PSMA } \\
\leq 10 \% \text { vs }>10 \%\end{array}$ \\
\hline \multicolumn{7}{|l|}{${ }^{a}$ Bone invasion } \\
\hline Present & $27(25 \%)$ & $17 \%$ & $-.20 ; p=.04$ & $10(37 \%)$ & $17(63 \%)$ & $p=.02$ \\
\hline Absent & $82(75 \%)$ & $39 \%$ & & $13(16 \%)$ & $69(84 \%)$ & \\
\hline \multicolumn{7}{|l|}{ Growth pattern (Perzin grade [21]) } \\
\hline Tubular (grade 1) & $46(42 \%)$ & $30 \%$ & $.12 ; p=.21$ & $10(22 \%)$ & $36(78 \%)$ & $p=.89$ \\
\hline Cribriform; < 30\% solid (grade 2) & $43(39 \%)$ & $33 \%$ & & $7(16 \%)$ & $36(84 \%)$ & $p=.32$ \\
\hline Solid (grade 3) & $20(18 \%)$ & $46 \%$ & & $6(30 \%)$ & $14(70 \%)$ & $p=.28$ \\
\hline \multicolumn{7}{|l|}{ Treatment } \\
\hline \multicolumn{7}{|l|}{ Adjuvant radiotherapy } \\
\hline Yes & $102(93 \%)$ & $33 \%$ & $.14 ; p=.15$ & $20(20 \%)$ & $82(80 \%)$ & $p=.23$ \\
\hline No & $8(7 \%)$ & $26 \%$ & & $3(38 \%)$ & $5(62 \%)$ & \\
\hline
\end{tabular}

a difference is considered statistically significant

15-60\%). Median PSMA expression and Spearman- $\rho$ correlation coefficients for all clinical parameters and pathological characteristics are listed in Table 1. Although no strong associations were found, Spearman- $\rho$ analysis revealed a significant association between PSMA expression and both tumour subsite and bone invasion. The minor salivary glands demonstrated less PSMA expression than the major salivary glands ( 21 vs. $50 \%$ median expression; $p<.01)$ and tumours invading bone showed less PSMA expression (17 vs. 39\% median expression; $p=.04$ ). However, tumour subsite and bone invasion are mutually strongly correlated (Pearson chi-square test $p<.01$ ) as all except 1 of the tumours invading bone originated from the minor salivary glands. Median PSMA expression did not differ between the subgroups of tumour site, pathological tumour stage (pT-stage) and Perzin grade. Furthermore, primary tumour PSMA expression medians of patients who developed a locoregional recurrence or
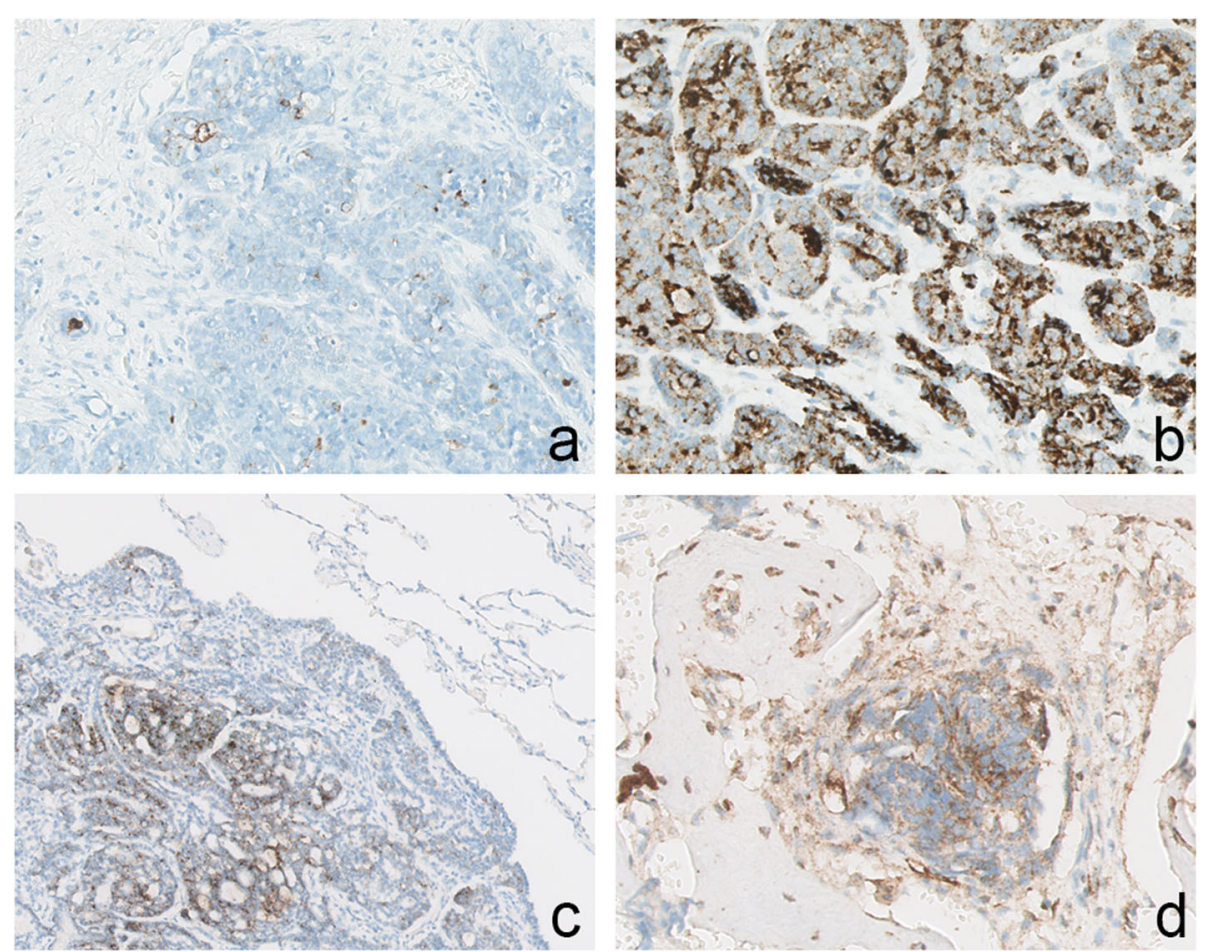

Fig. 1 Representative immunohistochemical PSMA expression in primary and metastatic AdCC showing variation in the number of positive staining tumour cells. a nasal cavity; 5\%; magnification 200x. b submandibular gland; 90\%; magnification 400x. c pulmonary metastasis; 70\%; magnification 200x. $\mathbf{d}$ bone metastasis; 5\%; magnification 200x 
Table 2 Follow-up

\begin{tabular}{|c|c|c|c|c|c|c|}
\hline & N (\%) & Median months; IQR & Median \% PSMA primary tumour & $\begin{array}{l}\text { PSMA } \\
0-10 \% \\
\end{array}$ & $\begin{array}{l}\text { PSMA } \\
>10 \%\end{array}$ & $\begin{array}{l}\mathrm{Chi}^{2} \text { PSMA } \\
\leq 10 \% \text { vs }>10 \%\end{array}$ \\
\hline Patients & 110 & & & 23 & 87 & \\
\hline Follow-up (median months; IQR) & & $57 ; 26-100$ & & $50 ; 13-96$ & $63 ; 27-110$ & \\
\hline \multicolumn{7}{|l|}{ Locoregional recurrence } \\
\hline Yes & $29(26 \%)$ & $39 ; 20-91$ & $40 \%$ & 9 & 20 & $p=.12$ \\
\hline No & $81(74 \%)$ & & $30 \%$ & 14 & 67 & \\
\hline \multicolumn{7}{|l|}{ Distant metastasis } \\
\hline Yes & $36(33 \%)$ & $33 ; 12-77$ & $38 \%$ & 7 & 29 & $p=.79$ \\
\hline No & $74(67 \%)$ & & $30 \%$ & 16 & 58 & \\
\hline \multicolumn{7}{|c|}{ aLocoregional recurrence-free @ 10 years } \\
\hline Yes & $84(76 \%)$ & & $35 \%$ & 14 & 70 & $p=.05$ \\
\hline No & $26(24 \%)$ & & $31 \%$ & 9 & 17 & \\
\hline
\end{tabular}

${ }^{a}$ difference is considered statistically significant

distant metastasis seem higher, but did not differ statistically from the distribution of those who remained diseasefree (Table 2).

\section{PSMA expression analysis in recurrent and distant metastatic AdCC}

Fifteen locoregional recurrent and 10 distant metastatic tissue samples could be retrieved from 18 patients. Nine of these 18 patients developed only locoregional recurrence(s), 6 patients developed only distant metastases and 3 patients developed both a recurrence and metastases. Distant metastatic tissues were lung (3 cases), leptomeningeal (3 cases), bone (1 case), liver (1 case), peritoneum (1 case) and skin (1 case). Positive PSMA expression was seen in 12/15 (80\%) recurrent and 9/10 (90\%) distant samples that ranged from 5 to $100 \%$. All together, the median PSMA expression of the recurrent samples was $60 \%$ (IQR 30-90\%) and of all metastatic samples $23 \%$ (IQR 10-55\%). When these were compared to the expression of their corresponding 18 primary tumours (40\% median expression; IQR 15-70\%), there was a (non-significant) tendency of increased expression in recurrences and decreased expression in distant metastases. Expression patterns of corresponding tumour samples are visualized in Supplementary Figure 1.

\section{Follow-up and survival analysis}

Details of follow-up and survival rates are summarized in Tables 2 and 3. Dichotomization of PSMA expression was carried out by plotting different ROC-curves for prediction of OS, DSS, RFS and MFS, which showed optimal cut-off points in a range of $8-12 \%$. Although the area under these curves was not sufficient to assume high sensitivity and specificity, for practical reasons a merged cut-off point of $10 \%$ PSMA expression was defined (graphs not shown). The $0-10 \%$ expression group was dominated by patients whose tumour was located in the minor salivary glands and, more specifically, in the nasal cavity, nasopharynx or maxillary sinus as revealed by post-hoc analysis $(p=.01$ and $p<.01$ respectively). Furthermore, in this group, relatively more tumours showed bone invasion $(p=.02)$ and relatively more patients were not recurrence-free 10 years after diagnosis $(p=.05)$. Despite half of the pT4b tumours expressed 0 $10 \%$ PSMA, this distribution did not reach statistical significance due to multiple testing ( $p=.04)$, see Table 1 . A multivariate Cox-proportional Hazard model with Firth's correction showed a significant relation between low PSMA expression (0-10\%) and worse 10-years RFS (HR 3.0, 95\% CI 1.1-8.5, $p=.04)$. A $>30 \%$ solid growth pattern / Perzin grade 3 (HR 3.7, 95\% CI 1.3-11.2, $p=.02$ ),

Table 3 Survival data

\begin{tabular}{|c|c|c|c|}
\hline & $\mathrm{N}$ affected & Median months & $\%$ survival \\
\hline \multicolumn{4}{|l|}{ Overall survival } \\
\hline 5-year OS & 25 & 36 & $77 \%$ \\
\hline 10-year OS & 29 & 41 & $74 \%$ \\
\hline \multicolumn{4}{|c|}{ Disease-specific survival } \\
\hline 5-year DSS & 17 & 41 & $85 \%$ \\
\hline 10-year DSS & 21 & 47 & $81 \%$ \\
\hline \multicolumn{4}{|c|}{ Disease-free survival } \\
\hline 5-year DFS & 36 & 20 & $67 \%$ \\
\hline 10-year DFS & 49 & 31 & $55 \%$ \\
\hline \multicolumn{4}{|c|}{ Locoregional recurrence-free survival } \\
\hline 5-year RFS & 19 & 27 & $83 \%$ \\
\hline 10-year RFS & 26 & 36 & $76 \%$ \\
\hline \multicolumn{4}{|c|}{ Metastatic-free survival } \\
\hline 5-year MFS & 26 & 22 & $76 \%$ \\
\hline 10-year MFS & 33 & 28 & $70 \%$ \\
\hline
\end{tabular}


tumour localization in the nasal cavity, nasopharynx or maxillary sinus (HR 41.7, 95\% CI 6.2-236.8, $p<.01$ ), and no postoperative radiotherapy (HR 5.1, 95\% CI 1.2-17.5, $p=.02$ ) were also significant prognosticators for locoregional recurrence within 10 years. Harrell's C-statistic of the predictive Cox-proportional Hazard model was 0.81, and without PSMA expression 0.79. PSMA expression was no significant contributor for the prediction of OS, DSS and MFS (multivariate data not shown).

\section{Discussion}

This is the first large cohort study describing PSMA expression in primary, recurrent and distant metastatic AdCC of the head and neck. Positive expression was seen in $94 \%$ of the primary tumours, $80 \%$ of recurrent tumours and $90 \%$ of the distant metastases, with PSMA expressed in 31, 60 and $23 \%$ of the tumour cells respectively. Primary tumour expression could not indicate disease progression and could not estimate expression levels in a recurrence or metastasis, although a tendency of respectively increase and decrease was observed. PSMA expression was not correlated to pathological stage and grade.

This is in contrast to PC in which high PSMA expression is correlated with prostate-specific antigen (PSA) recurrence and other prognostic factors which negatively affect survival such as tumour grade, pathological stage and castration resistance $[4,24]$. Multiple studies have shown that PSMA activates AKT and MAPK pathways promoting proliferation and survival of cancer cells, which may lead to an aggressive biological and clinical behaviour $[25,26]$. However, the currently presented inverse correlation of low primary tumour PSMA expression $\leq 10 \%$ as independent predictor of shortened RFS (HR 3.0; 95\% CI 1.1-8.5; $p=.04$ ) has also been described in other cancer types and might partly be explained by epigenetic silencing of the PSMA gene upon tumour progression [27].

Analogous to PC, AdCC demonstrates expression of PSMA in the epithelial tumour cells, while expression in different other tumours mainly concentrates in endothelial cells of tumour-associated neovasculature [3, 6, 28].

Of all primary PCs, 95\% show heterogeneous PSMA expression with on average $53 \pm 32 \%$ (mean \pm SD) positive tumour cells. Mean expression in regional lymph nodes and distant metastasis is more extensive $(72 \pm 36 \%$ and $92 \pm 10 \%$ respectively). Normal prostate tissue shows high PSMA expression in $100 \%$ of the samples $(77 \pm 32 \%$ positive cells), but with significantly less staining intensity than tumour tissue $[3,4]$.

In contrast, staining intensity in AdCC is relatively constant. Although it is known that major and minor salivary glands depict high tracer uptake on PSMA PET/CT, a comparison between expression intensity in normal salivary gland tissue and AdCC tumour tissue could not be made due to the lack of data on PSMA expression in non-pathologic salivary glands [29]. Comparing published PC data and our AdCC data, the present study concludes that similar to PC, $94 \%$ of primary AdCC expresses PSMA, but AdCC expression is more homogenous in a lower percentage of positive tumour cells.

Some points need to be addressed. Multiple Spearman- $\rho$ correlation analyses and KW nonparametric tests were carried out to analyse possible differences between a large amount of clinical parameters and tumour characteristics. False-positive findings might have been introduced, as adjustments to correct for multiple comparisons are not desirable in explorative studies and were therefore not applied. The results of these analyses should therefore be interpreted carefully [30].

By comparing the dichotomized groups of PSMA expression, it is noticed that a relatively large number of tumours in the low $\leq 10 \%$ PSMA expression group are located in the nasal cavity, nasopharynx or maxillary sinus, sites that are known to have a worse prognosis when compared to tumours originating from other subsites [11]. Notwithstanding the small sample sizes in these study subgroups, specifically the aforementioned tumour sites are, besides poor RFS, concordantly associated with poor DSS and OS (data not shown). Tumour stage $\mathrm{T} 4 \mathrm{~b}$ and bone invasion were also over-represented in the low PSMA expression $(\leq 10 \%)$ group. Although these parameters themselves are no independent predictors of RFS, tumour stage is strongly correlated with DSS and OS. Furthermore, tumour stage and bone invasion are mutually highly correlated (Pearson chi-square test $p<.01$ ) as all except one T4b tumour showed bone invasion. Moreover, bone invasion is significantly associated with tumour localization in the nasal cavity, nasopharynx or maxillary sinus. Tumours at these locations recur more often. These collinearities could be explained by delayed presentation of tumours from this subsite (that often involves the skull base), but may have confounded the results.

Another factor of debate is the limited discriminative strength of the $10 \%$ cut-off point. The above mentioned considerations are supported by the minimal increase of Harrell's C-statistic of the multivariate Cox-proportional Hazard model by 0.02 and therefore the additive value of PSMA to the prediction of RFS remains questionable.

A deep locoregional recurrence or growing distant metastases, for which conventional treatment options are no longer applicable due to functional irresectability or exceeded radiation limits, is a relevant problem in the management of AdCC. The limited experience with palliative chemotherapeutic agents and new initiatives with different targeted agents in this setting was recently reviewed [31]. The present results of high PSMA 
expression in primary, recurrent and metastatic tumour cells with limited spatial and temporal variability, as well as the uptake of PSMA ligand in recurrent and distant metastatic AdCC on PET/CT, could suggest a potential role for palliative targeted treatment with Lutetium-177PSMA [10, 32]. First large trials of this radionuclide treatment of metastatic castration-resistant PC show high response rates with low toxicity, improved quality of life and even prolonged OS [20,33]. Regarding AdCC, the high amount of associated grade I xerostomia (87\%) must be taken into account, as these patients usually have already been exposed to radiotherapy to the head and neck before [20].

\section{Conclusions}

This study shows unambiguous PSMA expression in a large cohort of primary, recurrent and metastatic AdCC of the head and neck. Expression was seen in 94\% of the primary tumours, which is analogous to PC except for the median lower number of positive tumour cells. In general, there was no relation between upregulated PSMA expression and pathological stage, tumour grade (growth pattern), the occurrence of locoregional recurrence or metastasis, and survival. Low primary tumour expression $\leq 10 \%$ is significantly associated with worsened RFS although its predictive value is limited. Of the recurrent and distant samples, respectively 87 and $90 \%$ were PSMA-positive, but staining could not be estimated based on primary tumour expression. This study provides encouraging supporting results that when other palliative systemic treatment options fail, PSMA-targeted imaging followed by experimental Lutetium-177-PSMA radionuclide therapy in AdCC, might be an alternative.

\section{Supplementary information}

Supplementary information accompanies this paper at https://doi.org/10. 1186/s12885-020-06847-9.

Additional file 1: Supplementary Figure 1. Differences in PSMA expression between primary, recurrent and metastatic AdCC in 18 patients, in order of PSMA expression of the primary tumour.

\begin{abstract}
Abbreviations
PSMA: Prostate-Specific Membrane Antigen; AdCC: Adenoid cystic carcinoma; PC: Prostate carcinoma; TMA: Tissue microarray; OS: Overall survival; DSS: Disease-specific survival; DFS: Disease-free survival; RFS: Recurrence-free survival; MFS: Metastatic-free survival; IQR: Interquartile range; Cl: Confidence interval; HR: Hazard ratio; KW: Kruskal-Wallis test; ROC: Receiver Operating Characteristic
\end{abstract}

\section{Acknowledgements}

None.

\section{Authors' contributions}

All authors read and approved the final manuscript. Study concepts: SMW. Study design: TKN, SMW. Data acquisition: TKN, MHV, SMW, LAS. Quality control of data and algorithms: TKN, MHV, LES, BdK, RvE, RdB. Data analysis, interpretation and statistical analysis: TKN, NPZ. Manuscript preparation: TKN, MHV. Manuscript editing and review: RvE, RdB, BdK, SMW, LAS, LES.

\section{Funding}

This research did not receive any specific grant from funding agencies in the public, commercial, or not-for-profit sectors.

\section{Availability of data and materials}

The datasets used and/or analysed during the current study are available from the corresponding author on reasonable request.

\section{Ethics approval and consent to participate}

For this study on residual tissue formal consent is not required. This was approved by both the institutional Medical and Biobank Research Ethics Committee, protocol numbers UMCU $16-564$ and 17-073 respectively. This is in accordance with the 1964 Helsinki declaration and its later amendments or comparable ethical standards.

\section{Consent for publication}

Not applicable.

\section{Competing interests}

The authors declare that they have no competing interests.

\section{Author details}

${ }^{1}$ Department of Head and Neck Surgical Oncology, UMC Utrecht Cancer Center, University Medical Center Utrecht, Heidelberglaan 100, P.O. Box 85500, Utrecht 3508, GA, The Netherlands. ${ }^{2}$ Department of Oral and Maxillofacial Surgery, University Medical Center Utrecht, Utrecht, The Netherlands. ${ }^{3}$ Department of Head and Neck Oncology and Surgery, Netherlands Cancer Institute/Antoni van Leeuwenhoek, Amsterdam, The Netherlands. ${ }^{4}$ Department of Oral and Maxillofacial Surgery, Amsterdam UMC, University of Amsterdam, Amsterdam, The Netherlands. ${ }^{5}$ Department of Pathology, Netherlands Cancer Institute/Antoni van Leeuwenhoek, Amsterdam, The Netherlands. ${ }^{6} J u l i u s$ Center for Health Sciences and Primary Care, University Medical Center Utrecht, Utrecht, The Netherlands.

${ }^{7}$ Department of Radiology and Nuclear Medicine, University Medical Center Utrecht, Utrecht, The Netherlands. ${ }^{8}$ Department of Pathology, University Medical Center Utrecht, Utrecht, The Netherlands.

Received: 3 October 2019 Accepted: 7 April 2020

Published online: 05 June 2020

\section{References}

1. Horoszewicz JS, Kawinsky EMG. Monoclonal antibodies to a new antigenic marker in epithelial prostatic cells and serum of prostatic cancer patients. Anticancer Res. 1987;7:927-35.

2. Israeli RS, Powell CT, Corr JG, Fair WR, Heston WD. Expression of the prostate-specific membrane antigen. Cancer Res. 1994;54:1807-11.

3. Wright GL, Haley C, Lou BM, Schellhammer PF. Expression of prostatespecific membrane antigen in normal, benign, and malignant prostate tissues. Urol Oncol Semin Orig Investig. 1995;1:18-28.

4. Ross JS, Sheehan CE, Fisher HAG, et al. Correlation of primary tumor prostate-specific membrane antigen expression with disease recurrence in prostate cancer. Clin Cancer Res. 2003;9:6357-62.

5. Afshar-Oromieh A, Hetzheim $H$, Kratochwil $C$, et al. The novel theranostic PSMA-ligand PSMA-617 in the diagnosis of prostate cancer by PET/CT: biodistribution in humans, radiation dosimetry and first evaluation of tumor lesions. J Nucl Med. 2015:56:1697-705.

6. Backhaus P, Noto B, Avramovic N, et al. Targeting PSMA by radioligands in non-prostate disease - current status and future perspectives. Eur I Nucl Med Mol Imaging. 2018;45(5):860-77. https://doi.org/10.1007/s00259-0173922-y. Epub 2018 Jan 15.

7. Wolf $\mathrm{P}$, Freudenberg $\mathrm{N}$, Bühler $\mathrm{P}$, et al. Three conformational antibodies specific for different PSMA epitopes are promising diagnostic and therapeutic tools for prostate cancer. Prostate. 2010;70:562-9.

8. Chang SS, Reuter VE, Heston WDW, Bander NH, Grauer LS, Gaudin PB. Five different anti-prostate-specific membrane antigen (PSMA) antibodies confirm PSMA expression in tumor-associated Neovasculature. Cancer Res. 1999:59:3192-8.

9. Silver DA, Pellicer I, Fair WR, Heston WD, Cordon-Cardo C. Prostate-specific membrane antigen expression in normal and malignant human tissues. Am Assoc Cancer Res. 1997;3:81-5. 
10. Klein Nulent TJW, van Es RJJ, Krijger GC, de Bree R, Willems SM, de Keizer B. Prostate-specific membrane antigen PET imaging and immunohistochemistry in adenoid cystic carcinoma-a preliminary analysis. Eur J Nucl Med Mol Imaging. 2017:44(10):1614-21. https://doi.org/10.1007/ s00259-017-3737-x. Epub 2017 Jun 7.

11. Spiro RH, Huvos AG, Strong EW. Adenoid cystic carcinoma of salivary origin. A clinicopathologic study of 242 cases. Am J Surg. 1974;128:512-20.

12. Netherlands Comprehensive Cancer Organisation, The Netherlands Cancer Registry. Incidence of invasive salivary gland cancer per year. 2015.

13. de Ridder M, Balm AJM, Smeele LE, Wouters MWJM, van BAC D. An epidemiological evaluation of salivary gland cancer in the Netherlands (1989-2010). Cancer Epidemiol. 2015;39(1):14-20. https://doi.org/10.1016/j. canep.2014.10.007. Epub 2014 Nov 15.

14. Bjørndal K, Krogdahl A, Therkildsen $\mathbf{M H}$, et al. Salivary gland carcinoma in Denmark 1990-2005: a national study of incidence, site and histology. Results of the Danish head and neck Cancer group (DAHANCA). Oral Oncol. 2011:47:677-82

15. Jang S, Patel PN, Kimple RJ, McCulloch TM. Clinical outcomes and prognostic factors of adenoid cystic carcinoma of the head and neck. Anticancer Res. 2017;37:3045-52.

16. Coca-Pelaz A, Rodrigo JP, Bradley PJ, et al. Adenoid cystic carcinoma of the head and neck - an update. Oral Oncol. 2015;51:652-61.

17. Hellquist H, Skalova A. Adenoid Cystic Carcinoma. Histopathol. Berlin Heidelberg: Salivary Gland., Springer; 2014. p. 221-60.

18. van Weert $S$, Reinhard $R$, Bloemena $E$, et al. Differences in patterns of survival in metastatic adenoid cystic carcinoma of the head and neck. Head Neck. 2017;39:456-63.

19. Ciccolallo L, Licitra L, Cantú G, Gatta G. Survival from salivary glands adenoid cystic carcinoma in European populations. Oral Oncol. 2009;45:669-74.

20. Hofman MS, Violet J, Hicks RJ, et al. [177Lu]-PSMA-617 radionuclide treatment in patients with metastatic castration-resistant prostate cancer (LuPSMA trial): a single-centre, single-arm, phase 2 study. Lancet Oncol. 2018;19(6):825-33. https://doi.org/10.1016/S1470-2045(18)30198-0. Epub 2018 May 8.

21. Perzin KH, Gullane $P$, Clairmont AC. Adenoid cystic carcinomas arising in salivary glands: a correlation of histologic features and clinical course. Cancer. 1978:42:265-82.

22. Heinze G, Dunkler D. Avoiding infinite estimates of time-dependent effects in small-sample survival studies. Stat Med. 2008;27:6455-69.

23. Harrell FEH, Lee KL, Mark DB. Multivariable prognostic models: issues in developing models, evaluating assumptions and adequacy, and measurin and reducing errors. Stat Med. 1996;15:361-87.

24. Wright GL, Mayer Grob B, Haley C, et al. Upregulation of prostate-specific membrane antigen after androgen-deprivation therapy. Urology. 1996;48: 326-34.

25. Perico ME, Grasso S, Brunelli M, et al. Prostate-specific membrane antigen (PSMA) assembles a macromolecular complex regulating growth and survival of prostate cancer cells "in vitro" and correlating with progression "in vivo.". Oncotarget. 2016;7:74189-202.

26. Kaittanis C, Andreou C, Hieronymus $\mathrm{H}$, et al. Prostate-specific membrane antigen cleavage of vitamin B9 stimulates oncogenic signaling through metabotropic glutamate receptors. J Exp Med. 2018;215:159-75.

27. Mhawech-Fauceglia P, Smiraglia DJ, Bshara W, et al. Prostate-specific membrane antigen expression is a potential prognostic marker in endometrial adenocarcinoma. Cancer Epidemiol Biomark Prev. 2008;17:571-7.

28. Salas Fragomeni RA, Amir T, Sheikhbahaei S, et al. Imaging of Non-Prostate Cancers Using PSMA-Targeted Radiotracers: Rationale, Current State of the Field, and a Call to Arms. J Nucl Med. 2018. https://doi.org/10.2967/jnumed. 117.203570

29. Klein Nulent TJW, Valstar MH, de Keizer B, et al. Physiologic distribution of PSMA-ligand in salivary glands and seromucous glands of the head and neck on PET/CT. Oral Surg Oral Med Oral Pathol Oral Radiol. 2018.

30. Rothman KJ. No adjustments are needed for multiple comparisons. Epidemiology. 1990;1:43-6.

31. Alfieri S, Granata R, Bergamini C, et al. Systemic therapy in metastatic salivary gland carcinomas: a pathology-driven paradigm? Oral Oncol. 2017;66:58-63.

32. Has Simsek D, Kuyumcu S, Agaoglu FY, Unal SN. Radionuclide therapy with 177Lu-PSMA in a case of metastatic adenoid cystic carcinoma of the parotid. Clin Nucl Med. 2019:44:764-6.

33. Rahbar K, Boegemann M, Yordanova A, et al. PSMA targeted radioligandtherapy in metastatic castration resistant prostate cancer after chemotherapy, abiraterone and/or enzalutamide. A retrospective analysis of overall survival. Eur J Nucl Med Mol Imaging. 2018;45:12-9.

\section{Publisher's Note}

Springer Nature remains neutral with regard to jurisdictional claims in published maps and institutional affiliations.
Ready to submit your research? Choose BMC and benefit from:

- fast, convenient online submission

- thorough peer review by experienced researchers in your field

- rapid publication on acceptance

- support for research data, including large and complex data types

- gold Open Access which fosters wider collaboration and increased citations

- maximum visibility for your research: over $100 \mathrm{M}$ website views per year

At $\mathrm{BMC}$, research is always in progress.

Learn more biomedcentral.com/submissions 